\title{
Nitrógeno amoniacal en aguas residuales domesticas utilizando sedimentador primario con diferentes condiciones hidráulicas, marcara 2019
}

\author{
Kiko Félix Depaz Celi \\ kdepazc@ucvvirtual.edu.pe \\ Universidad Cesar Vallejo
}

Martin Miguel Huamán Carranza mhuamanc@unasam.edu.pe Universidad Nacional Santiago Antúnez De Mayolo

Jeyson Andersonn Rosales Fajardo jrosalesf@unasam.edu.pe Universidad Nacional Santiago Antúnez De Mayolo

Patricia Laura Gamarra Tahua pgamarrat@unasam.edu.pe Universidad Nacional Santiago Antúnez De Mayolo

\section{RESUMEN}

Huaraz - Perú

El objetivo de investigación fue verificar y determinar variación de incremento o descenso del nitrógeno amoniacal en aguas residuales domesticas utilizando tanques sépticos, condicionando criterio de diseño (relación: largo/ancho) en climas andinos, Tuyu Ruri -Marcara.

Donde se empleó 3 tipos de tanques sépticos de relación 2.3, 2.7 y 3.3 y grupo control con criterio de diseño reglamentado de 2.0, midiendo concentraciones en aguas residuales de $11.8 \mathrm{mg} / \mathrm{l}$ para nitrógeno amoniacal, $19.5 \mathrm{mg} / \mathrm{l}$ para Nitrógeno total, $7.7 \mathrm{mg} / \mathrm{l}$ para nitrógeno orgánico y demanda bioquímica de oxígeno de $75 \mathrm{mg} / \mathrm{l}$ DBO, medido en épocas de lluvia y estiaje; donde se controló parámetros de operación como temperatura promedio de $17,9^{\circ} \mathrm{C}$ entre los 4 tanques sépticos, $\mathrm{pH}$, caudal de ingreso, turbiedad y tiempo de retención entre las unidades de análisis.

Se determinó incremento del nitrógeno amoniacal en aguas residuales domesticas no influenciadas por relación de superficie (largo: ancho). Por tanto, el incremento del nitrógeno amoniacal en aguas residuales domesticas no está influenciada por este criterio de diseño en tanques sépticos, pero se observó remoción promedio del nitrógeno amoniacal del $25.275 \%$ con generación promedio de lodos de 1568.575 1/año y tiempo de retención promedio de 0.22 días y velocidad de sedimentación 1.7 m/día.

Palabras clave: nitrógeno amoniacal; aguas residuales; andino. 


\title{
Ammonia nitrogen in domestic wastewater using primary sedimentator with different hydraulic conditions, mark 2019
}

\begin{abstract}
The research objective was to verify and determine the variation in the increase or decrease of ammonia nitrogen in domestic wastewater using septic tanks, determining design criteria (ratio: length / width) in Andean climates, Tuyu Ruri -Marcara.

Where 3 types of septic tanks of ratio 2.3, 2.7 and 3.3 were used and control group with regulated design criteria of 2.0, measuring concentrations in wastewater of $11.8 \mathrm{mg} / 1$ for ammoniacal nitrogen, $19.5 \mathrm{mg} / 1$ for total Nitrogen, $7.7 \mathrm{mg} / 1$ for organic nitrogen and biochemical oxygen demand of $75 \mathrm{mg} / 1 \mathrm{BOD}$, measured in times of rain and low water; where operating parameters such as average temperature of $17.9^{\circ} \mathrm{C}$ between the 4 septic tanks, $\mathrm{pH}$, inflow flow, turbidity and retention time between the analysis units were controlled.

Increase in ammonia nitrogen in domestic wastewater not influenced by surface ratio (length: width) was determined. Therefore, the increase in ammonia nitrogen in domestic wastewater is not influenced by this design criterion in septic tanks, but an average removal of ammonia nitrogen of $25.275 \%$ was observed with an average generation of sludge of $1568.5751 /$ year and average retention time. 0.22 days and sedimentation velocity $1.7 \mathrm{~m} /$ day.
\end{abstract}

Keywords: ammonia nitrogen; wastewater; andean.

Artículo recibido: 10. Junio. 2021 Aceptado para publicación: 16. Julio. 2021 Correspondencia: kdepazc@ucvvirtual.edu.pe Conflictos de Interés: Ninguna que declarar 


\section{INTRODUCCIÓN}

La investigación de basa en comprobar el comportamiento del nitrógeno amoniacal en el tratamiento de las aguas residuales domesticas donde se consideró antecedentes resaltantes del comportamiento del $\mathrm{NH} 4$, por ejemplo existen investigaciones donde resalta la importancia de identificar claramente los efectos inhibidores del amoníaco sindicalizado (FA) y el ácido nitroso sindicalizado (FNA) sobre el proceso de nitrificación, pues se conoce que Las concentraciones de FA y FNA, en lugar de las concentraciones totales de iones de amoníaco o nitrito inhiben la nitrificación (Anthonisen et al., 1976). Se considero este aspecto porque las aguas residuales de la localidad de Tuyu Ruri se generan en la zona rural andina de Ancash, Perú, donde se usan insumos agroindustriales que pueden generar este exceso y se infiltran dentro del alcantarillado sanitario.

Como la unidad a estudiar es un tratamiento primario se consideró tener el control al momento de la caracterización de las aguas residuales con sus parámetros de estudio como lo fue la DQO, DBO y NH3-N, en investigaciones previas se determinó que provienen principalmente de la extracción y lavado de carbón, la fabricación de materias primas químicas y productos químicos, la industria papelera y la industria de procesamiento de alimentos(Wang et al., 2016), para nuestro estudio se enfocó del nitrógeno amoniacal doméstico y afluentes mínimos que provienen de las parcelas agrícolas las más cercanas a la red de alcantarillado sanitario.

Los efluentes de aguas residuales que son sometidos a una digestión anaeróbica de los desechos orgánicos suelen contener altas concentraciones de amonio, fosfato, sólidos en suspensión y sustrato orgánico persistente. Por tanto, existe un interés creciente en este tratamiento debido a sus ventajas en términos energéticos y al hecho de que produce fertilizante para reciclar los nutrientes a las tierras agrícolas. (Salminen et al., 2001)

Así también como se conoce que el tanque séptico es un tratamiento anaeróbico de baja carga y se consideró que el amoníaco logre una estabilidad baja en comparación con los de un digestor de alta carga que se controla a temperatura $\left(55,70,85^{\circ} \mathrm{C}\right), \mathrm{pH}(\sin$ ajustar y pH 10) y tiempo de retención hidráulico (2-5 días). (Serna-Maza et al., 2014). Pero también es importante recordar que el principal problema de algunos procesos de digestión anaeróbica es la gran cantidad de amoníaco que se libera, especialmente cuando se implementa una alta digestión de sólidos (Benabdallah El Hadj et al., 2009), el 
amoniaco libre afecta la fermentación metanogénica sino también la concentración de iones amonio. Otras condiciones como la presencia de oxígeno o fenómenos anóxicos también aportar para la variación del comportamiento del nitrógeno amoniacal, como ocurre en un sistema de lodos activados donde se cuenta con un sedimentador, la eficiencia de eliminación de amonio se vería comprometida debido al bajo nivel de oxígeno disuelto (OD), el proceso de combinación de eliminación de nitrógeno a través de nitrito y carga filamentosa limitada se logró para mejorar la eliminación de nitrógeno y reducir el consumo de energía de aireación mediante el control de niveles bajos de OD (0,5-1,0 mgL-1).(Guo et al., 2013); Tomando en cuenta que también existen acumulación del amoniaco libre y la limitación del oxígeno, estas dos condiciones deben de ser también verificadas y controladas en campo pues puede darse una fuerte interdependencia entre estos dos parámetros como sustratos para la oxidación del amoníaco(Rongsayamanont et al., 2019), esta elación se propone como un parámetro de control para la nitrificación.

También se usan tanques sépticos con doble cámara y otros con compartimiento adicionales donde van materiales filtrantes donde se adhiere y forma la biofilm, convirtiéndose en una celda microbiana de cámara única continua, es aplicable a las aguas residuales que contienen una alta concentración de nitrógeno mediante un proceso de adaptación. (Kim et al., 2011), considerando que esta opción también es válida para la remoción de nitrógeno amoniacal.

Existe también evidencia que las concentraciones de nitrógeno amoniacal está relacionado con la cantidad de DQO presente en el agua residual, para eliminar la DQO de las aguas residuales con alto contenido de amoníaco con una relación de carbononitrógeno baja y regular las formas de nitrógeno en el efluente del proceso, en ese estudio se contempló que el alto contenido de $\mathrm{NH} 4+-\mathrm{N}$ redujo la abundancia de desnitrificantes y bacterias degradantes orgánicas, mientras que aumentó la de bacterias fijadoras de nitrógeno (Niu et al., 2021) pues muy a pesar de la poca concentración que se cuente en aguas residuales de zona rural, es importante considerar el posible incremento de estas concentraciones si no se dan las condiciones de control adecuado.

Otro parámetro importante es que, para la eliminación de nitrógeno amoniacal de las aguas residuales, se debe tener un control adecuado del pH (Rongwong \& Sairiam, 2020). En los tanques sépticos se controló de acuerdo a las dimensiones optabas en el diseño, por esta razón también se puede relacionar con lo que se obtiene en un tratamiento de 
lodos activados, pues se conoce que en el tanque de aireación donde se realiza el proceso con un reactor discontinuo secuencial que trata aguas residuales domésticas. Las eficiencias de remoción promedio de DQO y TN aumentaron de 93 y $68.21 \%$ a 97 y 74.20\% con el aumento en MLSS de 3.5-4.0 a 7.5-8.0 g / L (Tian et al., 2018)

Considerando también que cuando no se hace una buena remoción de compuestos de nitrógeno en tratamiento terciario o en la pre y post cloración, como es el caso de la presencia de nitrógeno amoniacal y otros consumidores importantes de cloro daría como resultado una menor formación de actividad anti estrogénica (Tang et al., 2014)

Así mismo se sabe que la biomasa generada por la síntesis de los nutrientes en el agua residual que contiene elementos como proteína $=32 \%$, carbohidratos $=11 \%$ y lípidos totales $=18 \%$, y se ha logrado con el tratamiento de aguas residuales eficiencia de remociones: demanda química de oxígeno $=59 \%$, nitrógeno amoniacal $=78 \%$, fósforo total $=16 \%$ (Rodrigues de Assis et al., 2020), por lo tanto, podemos asegurar que si existen remociones importantes para el nitrógeno.

\section{ESTRATEGIAS METODOLÓGICAS O MATERIALES Y MÉTODOS}

Todos los procedimientos empleados para la investigación se basan en la determinación del incremento o variación del nitrógeno amoniacal en las aguas residuales domesticas en un sedimentador primario está influenciado por las condiciones hidráulicas (largo y ancho) en climas andinos-Tuyu, Marcara.

Se realizó el dimensionamiento de los sedimentadores primarios (tanques sépticos) teniendo como valores constantes el caudal de diseño, el tiempo de retención y con las siguientes relaciones largo y ancho $(\mathrm{L} / \mathrm{B})=2.0,2.3,2.7,3.3$ para su dimensionamiento.

Tabla 1: Parámetros y dimensiones de los sedimentadores primarios

\begin{tabular}{|c|c|c|c|c|c|}
\hline \multirow{2}{*}{$\begin{array}{c}\text { DISEÑO DEL TANQUE SEPTICO (T1) } \\
\text { RELACION L/B }\end{array}$} & \multicolumn{4}{|c|}{ RELACION L/B } & \multirow{2}{*}{ UND } \\
\hline & 1.96 & 2.30 & 2.73 & 3.30 & \\
\hline \multicolumn{6}{|l|}{ VOLUMEN DE SEDIMENTACION (Vs) (m3) } \\
\hline $\mathrm{Vs}=\mathrm{Q}(\mathrm{m} 3 / \mathrm{d}) * \mathrm{PR}(\mathrm{d})$ & 0.30 & 0.30 & 0.30 & 0.30 & $\mathrm{~m}^{3}$ \\
\hline 9.- VOLUMEN MINIMO DE NATAS (m3) & 0.082966667 & 0.0829667 & 0.0829667 & 0.0829667 & $\mathrm{~m}^{3}$ \\
\hline 10.- VOLUMEN DE Vs + Vd & 0.41 & 0.41 & 0.41 & 0.41 & $\mathrm{~m}^{3}$ \\
\hline Dimensionamiento de tanque séptico asumimos altura útil & $\mathrm{H}=0.50$ & 0.50 & 0.50 & 0.50 & $\mathrm{~m}$ \\
\hline Área útil & 0.830 & 0.830 & 0.830 & 0.830 & $\mathrm{~m}^{2}$ \\
\hline Relación L/B & 1.96 & 2.30 & 2.73 & 3.30 & \\
\hline Lovitud uाI (IiI) & $\mathrm{L}=1.27$ & 1.38 & 1.50 & 1.65 & $\mathrm{~m}$ \\
\hline Ancho útil (m) & $B=0.65$ & 0.60 & 0.55 & 0.50 & $\mathrm{~m}$ \\
\hline
\end{tabular}




\section{5.- DIMENCIONAMIENTO FINAL}

\begin{tabular}{lllllll} 
Altura total & $(\mathrm{m})$ & 0.75 & 0.75 & 0.75 & 0.75 & $\mathrm{~m}$ \\
Largo & $(\mathrm{m})$ & 1.27 & 1.38 & 1.50 & 1.65 & $\mathrm{~m}$ \\
Ancho & $(\mathrm{m})$ & 0.65 & 0.60 & 0.55 & 0.50 & $\mathrm{~m}$ \\
\hline
\end{tabular}

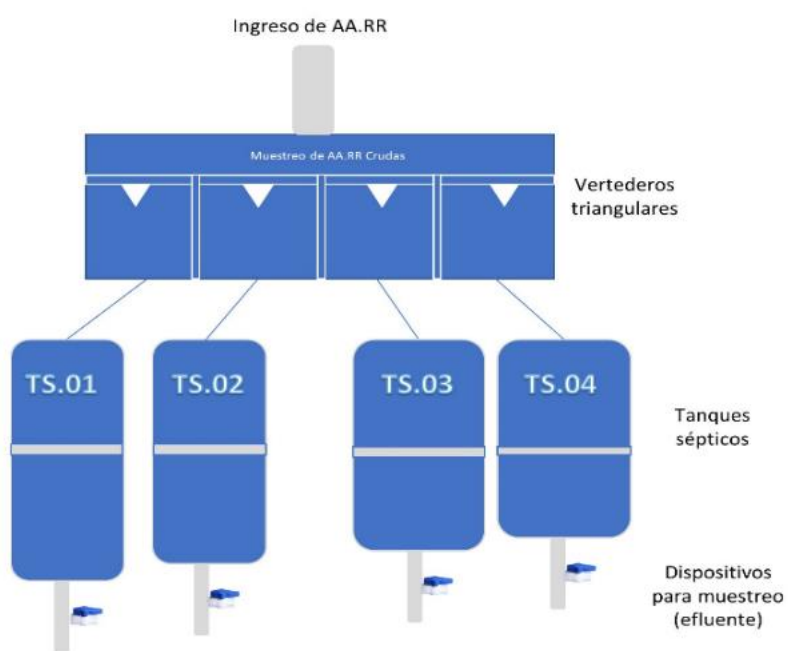

Figura 1: Esquema de instalación de piloto

\section{Puntos de monitoreo}

Agua residual cruda (afluente), se tomó en la entrada a los sedimentadores primarios, se ubicó en la unidad de repartición de caudal asegurándonos una zona donde no interfieran solidos de gran tamaño, esta unidad se encuentra después de una unidad de cribado.

El agua residual tratada (efluente), se colocó un dispositivo de salida donde se colocó dispositivos de salida (válvula de paso) que se instalaron a salida de cada tanque séptico, estos se implementaron para una mayor facilidad de la toma de muestras.

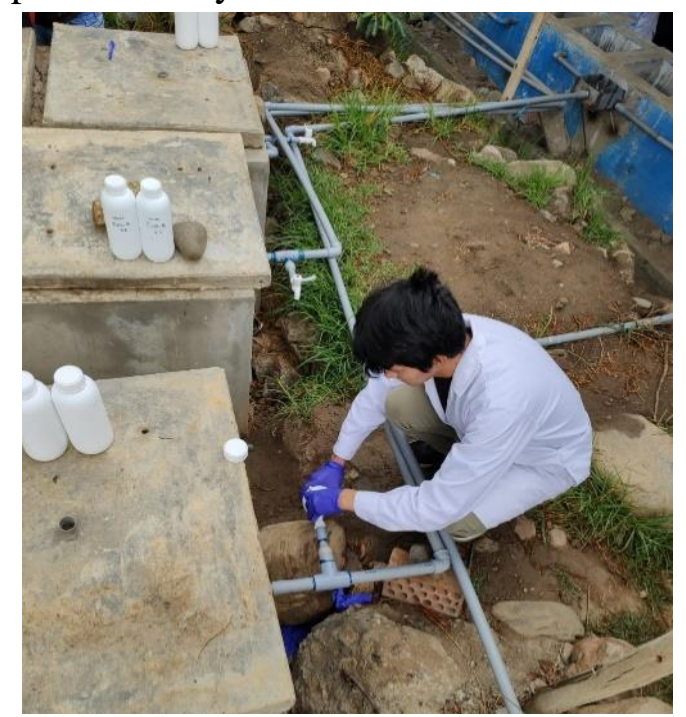

Ilustración 1: Toma de muestras en los pilotos. 
Los análisis fueron realizados en el laboratorio del centro experimental de Tuyu Ruri que se encuentra a $30 \mathrm{~m}$ aproximadamente de la zona de monitoreo, siguiendo los protocolos adecuados y estandarizados.(Federation \& Association, 2005).

Se monitorearon los siguientes parámetros en el agua residual cruda (afluente) y en el agua residual tratada (efluente), tomando en todos los casos muestras simples

- Nitrógeno amoniacal

- $\mathrm{pH}$

- Temperatura

- Oxígeno disuelto (solo en el afluente)

En la etapa de procesamiento y análisis los datos que se obtuvieron durante la caracterización de la muestra, para ello se aplicaron el uso de gráficos de cajas y bigotes, intervalos y puntos con un intervalo de confianza del $95 \%$ y el método estadístico annova para datos paramétricos y kurskal y walis para datos no paramétricos para identificar las diferencias entre los grupos de muestreo e interpretar el comportamiento de cada sedimentador primario en el proceso de tratamiento de las aguas residuales.

El análisis de varianza (Anova) es la técnica central en el análisis de datos experimentales paramétricos. La idea general de esta técnica es separar la variación total en las partes con las que contribuye cada fuente de variación en el experimento. En el caso del DCA se separan la variabilidad debida a los tratamientos y la debida al error. Cuando la primera predomina "claramente" sobre la segunda, es cuando se concluye que los tratamientos tienen efecto, o dicho de otra manera, las medias son diferentes. Cuando los tratamientos no dominan contribuyen igual o menos que el error, por lo que se concluye que las medias son iguales.(Ulibarrie et al., 2019)

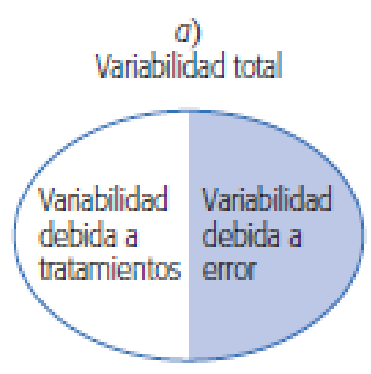

No hay efecto de tratarniento

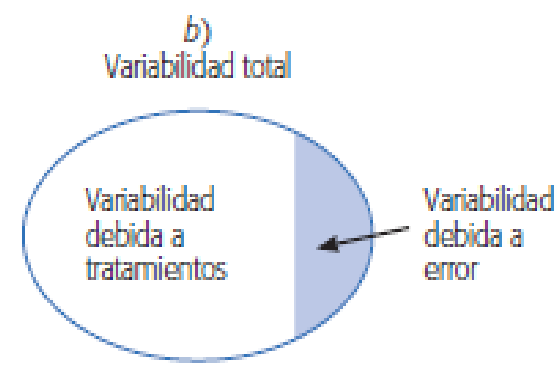

St hay efecto de tratamiento

Figura 2: Variabilidad según efectos de tratamiento 


\section{RESULTADOS Y DISCUSIÓN}

Se realizó un balance adecuado de los datos considerando primero conocer la variación de los flujos y caudales que se colocaron en el piloto, el agua residual ingresaba de forma continua con inspección diaria para evitar un inadecuado funcionamiento del sistema.

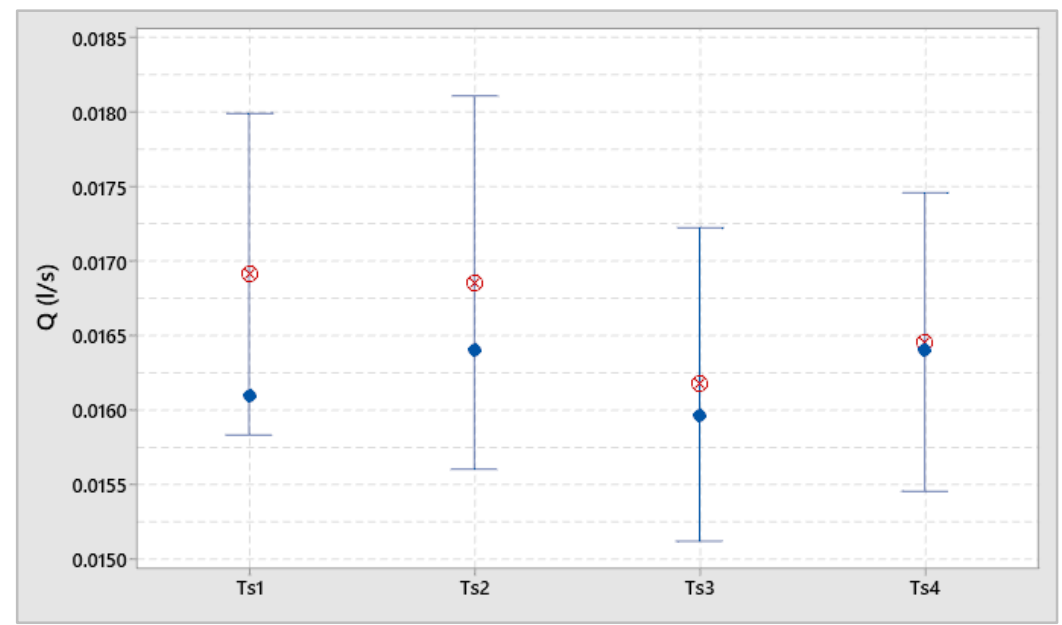

Figura 3: Variación de caudal de ingreso al piloto de tratamiento

Se observó intervalos de confianza del caudal de ingreso a los tanques sépticos, se observó caudales promedio con los valores de $0.0169,0.0168,0.01617,0.016451 / \mathrm{s}$, medianas de 0.3, 0.5, 0.2, 0.3, 0.2 para una toma de muestra en 5 fechas monitoreadas después del arranque de operación del piloto.

Los $\mathrm{pH}$ se tomaron medias todos los días desde el arranque y durante los días de monitoreo el cual resulto un $\mathrm{pH}$ promedio de 7.585, considerando también que hubo incrementos en las aguas residuales hasta un máximo de 7.62, lo cual evidenció que no afectaría nuestro tratamiento y procesos de remoción del $\mathrm{NH}_{3}-\mathrm{N}$

Así mismo se pudo demostrar que la variación de temperatura encontrada en todas las fechas de muestreo desde la puesta en marcha y el monitoreo del tratamiento tuvo valores máximos de $18.57^{\circ} \mathrm{C}$ y valor mínimo de $17.72^{\circ} \mathrm{C}$, se observó que en todos los tanques analizados la variación es mínima.

Una vez que se verifico el control el caudal, temperatura y $\mathrm{pH}$ en las unidades pilotos se realizó la medición de las concentraciones de Nitrógeno amoniacal $\mathrm{NH}_{3}-\mathrm{N}$, tanto en el afluente y efluente de los tanques sépticos, los cuales nos dieron los siguientes reportes: 

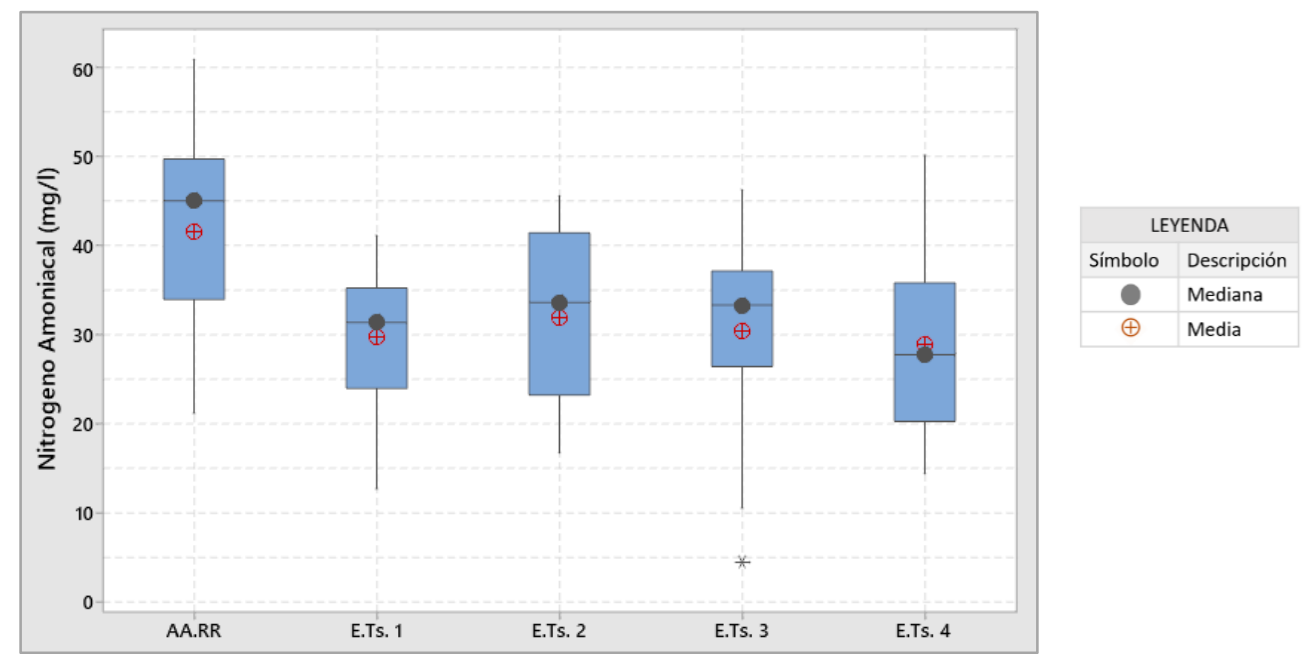

Figura 4: Intervalos de concentración de Nitrógeno Amoniacal

En la figura 4 de cajas y bigotes podemos observar valores promedio para el Nitrógeno amoniacal de $41.65,29.74,31.85,30.47,28.84 \mathrm{mg} / \mathrm{l}$, en cada tanque piloto evaluado comparado con la concentración de ingreso y como vario en el tiempo de proceso medido, que fue de 8 semanas, también se definió los valores máximos de 60.9, 41.12, 45.60, 46.27, $50.14 \mathrm{mg} / \mathrm{l}$ y valores mínimos de 21.19, 12.66, 16.76, 4.38, $14.42 \mathrm{mg} / \mathrm{l}$ respectivamente para los tanques $1,2,3,4$. Se observa un valor atípico para el valor mínimo obtenido en el tanque 3 esto se debe a que el ingreso del caudal era interrumpido debido a obstrucciones de las unidades por sólidos.

Para comprobar la influencia de los tanques primarios que fueron modificados en largo y ancho si guardan relación con remoción del nitrógeno amoniacal se estudió la relación de concentración final sobre inicial $\left(\mathrm{C} / \mathrm{C}_{\mathrm{o}}\right)$ como se muestra a continuación:

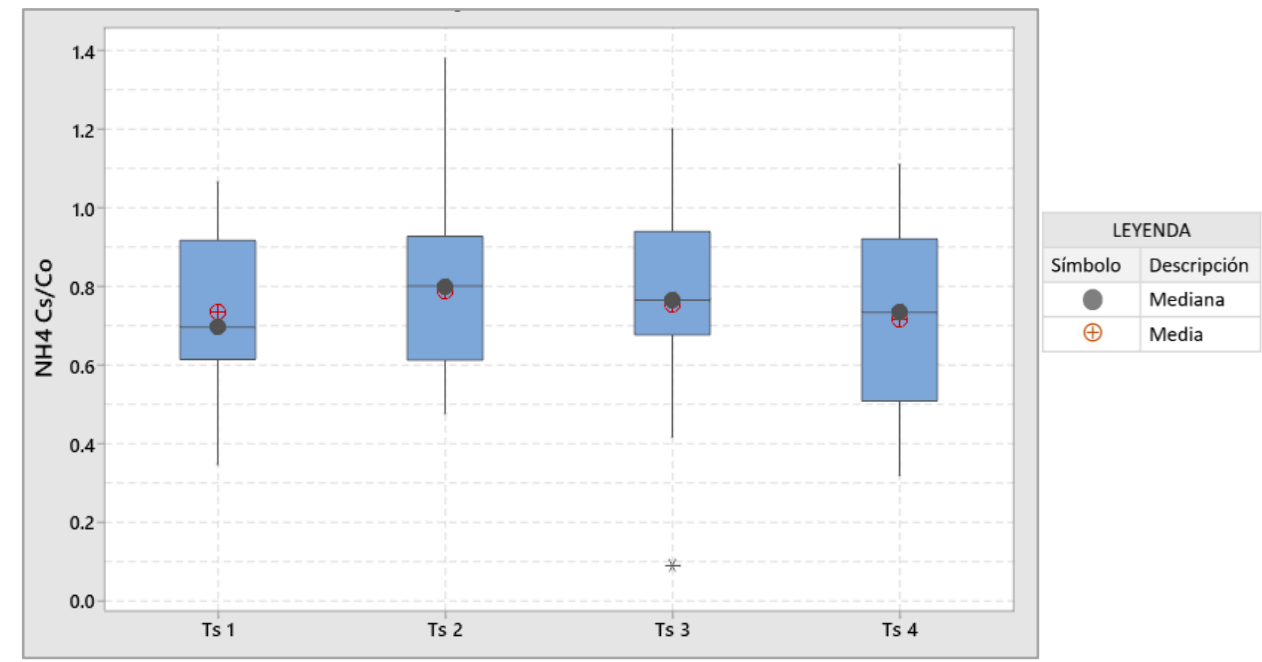

Figura 5: relación de concentraciones de $\mathrm{NH}_{3}-\mathrm{N}$ en los pilotos de tratamiento. 
En la figura 5 se observa los valores de la relación entre el efluente y afluente de los tanques sépticos (C/Co), se observó valores promedio de 0.7341, 0.7895, 0.7548, 0.7163, medianas de $0.68,0.80,0.77,0.73$, valores mínimos de $0.201,0.476,0.09,0.319$ y valores máximos de 1.0646, 1.3805, 1.2015, 1.110 para los tanques sépticos 1, 2, 3, 4 respectivamente. Además, se observó un valor atípico en el tanque 3 que corresponde a un valor fuera de lo común que representa de como variando de forma brusca el ingreso con respecto al de salida y esa variación fue mínima, esto principalmente debido a las obstrucciones de las unidades de ingreso y variación del caudal por ende de la carga.

También como un criterio de diseño y control de los sedimentadores que se estudiaron se comprobó el promedio de tiempo de retención de los tanques sépticos respectivamente con los valores de remoción del nitrógeno amoniacal observándose relaciones de tiempo de retención 5.04, 5.16, 5.352, 5.208 horas con valores de remoción de 29.2\%, 24\%, $21.4 \%, 26.5 \%$ respectivamente para los tanques $1,2,3,4$.

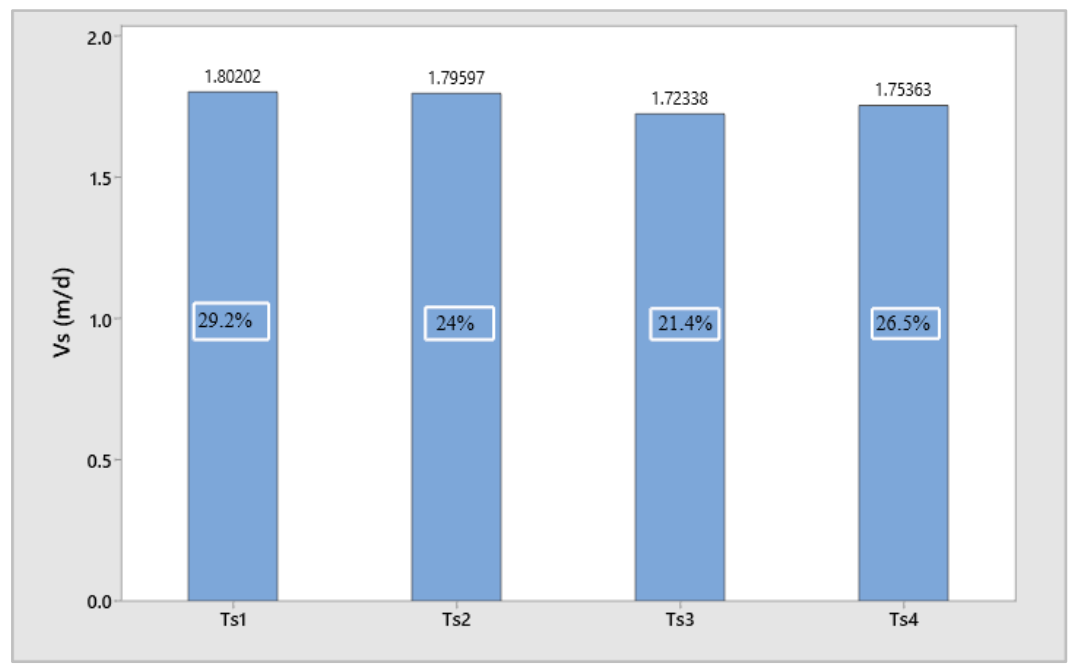

Figura 6: Comparación de velocidad de sedimentación vs eficiencia de remoción.

En la fig.06, se observa la variación de eficiencias y las tasas de sedimentación encontradas en cada uno de los tanques, pues como se explica esta investigación del piloto se basa en tanques primarios que son diseñados para la remoción de elementos suspendidos y que también afectan a cierta medida en la variación concentraciones de nitrógeno amoniacal de barras podemos observar la velocidad de sedimentación promedio de solidos en los tanques sépticos con valores de 1.802, 1.795, 1.723, 1.753 con valores de remoción de nitrógeno amoniacal de 29.2\%, 24\%, 21.4\%, 26.5\% respectivamente para los tanques $1,2,3,4$. 
Con los resultados ya medidos se comprobó con el método estadístico nuestro estudio de saber si existía alguna relación de los tanques sépticos con la remoción de Nitrógeno amoniacal en los sedimentadores, se identificó que no tiene relación con las condiciones hidráulicas en relación con la variación de la relación largo: ancho de los tanques sépticos (todas las medias son iguales), con un nivel de significancia: $\alpha=0.05$, contando la regla decisión: p - valor (sig.) >0,05

Tabla 2: análisis estadístico para remoción de Nitrógeno Amoniacal

\begin{tabular}{lrrrrrr}
\hline Fuente & GL & SC Ajust. & MC Ajust. & Valor F & Valor p & F critico \\
\hline Factor & 3 & 0.04745 & 0.007127 & 0.31 & 0.821 & 2.76 \\
Error & 60 & 3.10767 & 0.041354 & & & \\
\hline Total & 63 & 3.15512 & & & & \\
\hline
\end{tabular}

En la Tabla 2, podemos observar el p - valor (Sig.) alcanzado de 0.821 siendo mayor a 0,05 . Por lo tanto, se procede a validar con el procedimiento estadístico que el estudio realizado para la remoción de Nitrógeno amoniacal en los sedimentadores no tiene relación con las condiciones hidráulicas de los sedimentadores (relación larga/ancho de los tanques sépticos).

El comportamiento de las condiciones hidráulicas en relación con el caudal, no dieron los resultados mostrados con valores promedios de $0.0169 \mathrm{l} / \mathrm{s}$ para el afluente y 0.0169 , $0.0168,0.01617,0.01645 \mathrm{l} / \mathrm{s}$, para el efluente de cada tanque séptico, también se consideró que caudales mínimos de 0.0115, 0.0082, 0.0082, $0.0102 \mathrm{~L} / \mathrm{s}$ que se midieron en todo el tiempo de la experimentación y caudales máximos de 0.031, 0.034, 0.029, $0.028 \mathrm{~L} / \mathrm{s}$ para los tanques 1, 2, 3,4 respectivamente. Por otro lado, también se realizó la comprobación estadística de la variación de caudal logrando una evidencia con un resultado de p - valor de 0.117 que nos demuestra que el caudal de ingreso a los tanques sépticos tiene variación mínima y no significativa para el experimento. Con estos datos como referencia se puede afirmar que no existe significancia en la remoción de nitrógeno amoniacal, coincidiendo así en la evaluación de un sistema de tratamiento doméstico para reúso de agua residual se concluyó finalmente que los resultados demostraron que el sistema presentó un buen desempeño al aplicar los dos caudales $(0.2 \mathrm{~m} 3 / \mathrm{d}$ y $0.4 \mathrm{~m} 3 / \mathrm{d})$, pero la calidad del efluente que se obtuvo al aplicar el menor $(\mathrm{N}-\mathrm{NH} 4+\cdot \leq 3.77 \pm 3.3 \mathrm{mg} / \mathrm{L})$ cumplió para los parámetros evaluados con la norma mexicana (NOM-003-ECOL- 
1997).(Garzón Zúñiga et al., 2016) En la investigación el propósito de medir el caudal era evaluar las condiciones de ingreso de agua residual a cada tanque séptico afín de proporcionar las mimas condiciones para el experimento en cada tanque séptico y con la información presentada se afirma que el caudal de ingreso a cada tanque debe ser homogéneo para poder evaluar adecuadamente el comportamiento del NH4. Es preciso señalar que los intervalos del caudal nos expresan la variación drástica que se tiene del caudal, esto debido a que se realizaron muestreos diarios puntuales y por ser una zona rural los vertederos al ser de dimensiones pequeñas pueden influir en el caudal de ingreso, al tener que depender de la precisión humana de corte en las placas empleadas y ser unidades de pequeña dimensión.

\section{CONCLUSIÓN O CONSIDERACIONES FINALES}

Se determinó que el incremento del nitrógeno amoniacal en las aguas residuales domesticas no están influenciadas por la relación de superficie (largo: ancho) de los tanques sépticos.

Se realizo la caracterización de las aguas residuales observándose valores para las aguas residuales de 11,8 mg/l para el nitrógeno amoniacal, 19,5 mg/l para el Nitrógeno total, 7,7 mg/l para el nitrógeno orgánico y una demanda bioquímica de oxígeno de $75 \mathrm{mg} / \mathrm{l}$ DBO.

La temperatura en los tanques sépticos tuvo variación mínima entre los tanques sépticos y teniendo un promedio general de $17,9^{\circ} \mathrm{C}$ entre los 4 tanques sépticos valor que se encuentra dentro del rango para el desarrollo de proceso biológicos, el $\mathrm{Ph}$ presento una variación mínima entre tanques con un valor promedio general de 7,5 que se encuentra en el rango para el desarrollo de los procesos biológicos, el caudal de ingreso a todas las unidades no muestra variación significativa por lo que podemos afirmar que el caudal de ingreso fue homogéneo durante la investigación, la turbiedad presento una variación mínima entre grupos teniendo un valor promedio de remoción del $63 \%$ siendo el mejor el tanque numero 3 con una eficiencia del $65 \%$ lo que nos demuestra la funcionabilidad de la unidad de tratamiento al ser un sedimentador primario. El tiempo de retención presento una variación mínima con un valor promedio general de 5.19 horas (0.22 días). El oxígeno disuelto promedio fue de 4,5 mg/l que es determinante para la remoción del nitrógeno amoniacal. 
El Comportamiento del nitrógeno amoniacal durante el experimento presento una tendencia a la remoción de este contaminante con valores promedio de remoción del $25.275 \%$ con una generación promedio de lodos de 1568.575 1/año con un tiempo de retención promedio de 0.21 días y una velocidad de sedimentación de 1.7 m/día.

\section{LISTA DE REFERENCIAS}

Anthonisen, A. C., Loehr, R. C., Prakasam, T. B. S., \& Srinath, E. G. (1976). Inhibition of nitrification by ammonia and nitrous acid. Journal of the Water Pollution Control Federation, 48(5), $835-852$. https://www.scopus.com/inward/record.uri?eid=2-s2.00016949502\&partnerID=40\&md5=9fcce778d4f9ac07204f96ddefc 86492

Benabdallah El Hadj, T., Astals, S., Galí, A., Mace, S., \& Mata-Álvarez, J. (2009). Ammonia influence in anaerobic digestion of OFMSW. Water Science and Technology, 59(6), 1153-1158. https://doi.org/10.2166/wst.2009.100

Federation, W. E., \& Association, A. P. H. (2005). Standard methods for the examination of water and wastewater. American Public Health Association (APHA): Washington, DC, USA.

Garzón Zúñiga, M. A., González Zurita, J., \& García Barrios, R. (2016). Evaluación de un sistema de tratamiento doméstico para reúso de agua residual. Revista Internacional de Contaminación Ambiental, 32(2), 199-211.

Guo, J., Peng, Y., Yang, X., Gao, C., \& Wang, S. (2013). Combination process of limited filamentous bulking and nitrogen removal via nitrite for enhancing nitrogen removal and reducing aeration requirements. Chemosphere, 91(1). https://doi.org/10.1016/j.chemosphere.2012.12.004

Kim, H. W., Nam, J. Y., \& Shin, H. S. (2011). Ammonia inhibition and microbial adaptation in continuous single-chamber microbial fuel cells. Journal of Power Sources, 196(15). https://doi.org/10.1016/j.jpowsour.2011.03.061

Niu, J., Feng, Y., Wang, N., Liu, S., Liang, Y., Liu, J., \& He, W. (2021). Effects of high ammonia loading and in-situ short-cut nitrification in low carbon-nitrogen ratio wastewater treatment by biocathode microbial electrochemical system. Science of the Total Environment, 755. https://doi.org/10.1016/j.scitotenv.2020.142641

Rodrigues de Assis, L., Calijuri, M. L., Assemany, P. P., Silva, T. A., \& Teixeira, J. S. (2020). Innovative hybrid system for wastewater treatment: High-rate algal ponds 
for effluent treatment and biofilm reactor for biomass production and harvesting. Journal of Environmental Management, 274. https://doi.org/10.1016/j.jenvman.2020.111183

Rongsayamanont, C., Khan, E., \& Limpiyakorn, T. (2019). Dissolved oxygen/free ammonia (DO/FA) ratio manipulation to gain distinct proportions of nitrogen species in effluent of entrapped-cell-based reactors. Journal of Environmental Management, 251. https://doi.org/10.1016/j.jenvman.2019.109541

Rongwong, W., \& Sairiam, S. (2020). A modeling study on the effects of pH and partial wetting on the removal of ammonia nitrogen from wastewater by membrane contactors. Journal of Environmental Chemical Engineering, 8(5). https://doi.org/10.1016/j.jece.2020.104240

Salminen, E., Rintala, J., Härkönen, J., Kuitunen, M., Högmander, H., \& Oikari, A. (2001). Anaerobically digested poultry slaughterhouse wastes as fertiliser in agriculture. $\quad$ Bioresource $\quad$ Technology, 78(1), 81-88. https://doi.org/10.1016/S0960-8524(00)00160-7

Serna-Maza, A., Heaven, S., \& Banks, C. J. (2014). Ammonia removal in food waste anaerobic digestion using a side-stream stripping process. Bioresource Technology, 152 $307-315$. https://doi.org/https://doi.org/10.1016/j.biortech.2013.10.093

Tang, X., Wu, Q. Y., Du, Y., Yang, Y., \& Hu, H. Y. (2014). Anti-estrogenic activity formation potential assessment and precursor analysis in reclaimed water during chlorination. Water Research, 48(1). https://doi.org/10.1016/j.watres.2013.10.009

Tian, X., Guo, S., Xu, K., Zhao, C., Wang, X., \& Bian, D. (2018). Effect of mixed liquor suspended solids (MLSS) on simultaneous nitrification and denitrification in a sequencing batch reactor. IOP Conference Series: Earth and Environmental Science, 186(3). https://doi.org/10.1088/1755-1315/186/3/012041

Ulibarrie, N., Beltramini, L., Defagot, V., Bourquen, N., \& Andrés, F. (2019). Utilización del Diseño de Experimentos para la Optimización de Mezclas. Revista Tecnología y Ciencia, O(36 SE-Artículos). https://doi.org/10.33414/rtyc.36.11-21.2019

Wang, W., Liu, X., Wang, Y., Guo, X., \& Lu, S. (2016). Analysis of point source pollution and water environmental quality variation trends in the Nansi Lake basin 
from 2002 to 2012. Environmental Science and Pollution Research, 23(5). https://doi.org/10.1007/s11356-015-5625-X 